\title{
Spanning Tree Game as Prim Would Have Played*
}

\author{
András London ${ }^{a}$ and András Pluhár ${ }^{a}$
}

\begin{abstract}
In this paper, we investigate special types of Maker-Breaker games defined on graphs. We restrict Maker's possible moves that resembles the way that was introduced by Espig, Frieze, Krivelevich and Pedgen [9]. Here, we require that the subgraph induced by Maker's edges must be connected throughout the game. Besides the normal play, we examine the biased and accelerated versions of these games.
\end{abstract}

Keywords: positional games, spanning tree, biased games

\section{Introduction}

In a positional game two players play on a hypergraph $\mathcal{H}=(V(\mathcal{H}), E(\mathcal{H}))$, where $E(\mathcal{H})$ is usually referred as the family of winning sets. The players take turns in claiming vertices of $V(\mathcal{H})$ that was not claimed previously. In the Maker-Maker version a player wins by claiming every elements of some edge $A \in E(\mathcal{H})$ first. In the Maker-Breaker version Maker wins by claiming all elements of an edge, while Breaker wins if he can prevent Maker's win. Note that a Maker-Maker game may end in a draw, while only one of the players can win a Maker-Breaker game. The players may take more than one elements in a turn; we call it an $(\mathcal{H}, a, b)$-game if the first player takes $a$ and the second takes $b$ elements. If $a=b>1$ then it is an accelerated game, otherwise we call it a biased game. For a deeper introduction to positional games, we refer to Beck [4].

In graph games the set $V(\mathcal{H})$ is usually the edge set of a fixed graph $G$, mainly $G=K_{n}$, and $E(\mathcal{H})$ is a graph property. That is, Maker's goal is to build a particular structure (e.g. a spanning tree, a $K_{3}$ or a Hamiltonian cycle) within his own edges, while Breaker tries to prevent this. For some essential results in positional games, see e.g. [2, 3, 4]. Here we start with the classical Shannon's switching game, which is a Maker-Breaker game on the edge set of a connected graph $G$, and Maker wins

*This work was partially supported by the National Research, Development and Innovation Office - NKFIH, SNN-117879. The first author was supported by the project "Integrated program for training new generation of scientists in the fields of computer science", no EFOP-3.6.3-VEKOP16-2017-0002. The project was supported by the European Union and co-funded by the European Social Fund.

${ }^{a}$ Department of Computer Science, University of Szeged

E-mail: \{london,pluhar\}@inf .u-szeged.hu 
by taking the edges of a spanning tree. The outcome of this game is characterized by Lehman's theorem [12] stating that Maker wins (as a second player) if and only if the graph contains two edge-disjoint spanning trees.

Since the complete graph $K_{n}$ contains at least two disjoint spanning trees for $n \geq$ 4 , Shannon's switching game is trivial in this case. To make the game interesting for $K_{n}$, Chvátal and Erdős [7] introduced the $(1: b)$ biased version. The outcome is a monotone function of $b$ in a sense that if Maker wins for a value $b$, and $b^{\prime}<b$ then Maker also wins the $\left(1: b^{\prime}\right)$-game. Similarly, if Breaker wins the $(1: b)$ game, and $b<b^{\prime}$, then Breaker wins the $\left(1: b^{\prime}\right)$-game as well. Therefore they gave bounds on $b_{0}$, the smallest value for which Breaker wins. This turned out to be $b_{0}=\Theta(n / \log n)$, which can be considered from another viewpoint. If the two players take their selection randomly in a $(a, b)$-game, then the graph consisting of Maker's edges will be similar to an element of $G(n, p)$, where $p=a /(a+b)$. However, $p=\log n / n=\Theta(1 /(1+b))$ is the threshold for connectivity, see [6]. Hence one may say the perfect and random plays result in the same outcome. This probabilistic intuition, or Erdös paradigm gives a deep insight to a game, and turned out to be true for several cases [3, 4, 5], while it is also informative when it fails [1].

Epsig et al. [9] brought fresh ideas to the connectivity type of games by introducing Walker-Breaker game and PathWalker-Breaker game. Walker, being located on a vertex $x$, may claim an edge $e=(x, y)$ if $e$ has not been taken by Breaker before. Upon doing this, his location is changed to $y$. PathWalker is even more restricted; he is allowed to visit a vertex only once. For Breaker's moves, there are no restrictions. Walker and PathWalker wants to visit as many vertices of $G$ as possible. It was shown that Walker (and even PathWalker) reaches at least $n-2$ vertices of $K_{n}$ for large $n$. In the $1: b$-game the number of vertices that can be visited by PathWalker falls into the interval $\left[n-c_{1} \log n, n-c_{2} \log n\right]$, where the values of $c_{2}<c_{1}$ depend on only $b$.

In this study, motivated by the previous approach and some classic problems, we define new versions of Shannon's switching game. These are Maker-Breaker games where Maker's goal is to build a connected spanning subgraph of a graph $G$ such that in any moment of the game the subgraph consisting of Maker's edges is connected. We call this type of game the PrimMaker-Breaker game, referring to the execution of Prim's algorithm. Note that Prim's algorithm [16] finds a (minimal) spanning tree in a weighted undirected graph by keeping the subgraph of the already selected edges connected, in contrast to Kruskal's algorithm [11], which does not have this property. As a first step, we give a characterization for the $(1: 1)$ unbiased game ( i.e. each player takes one edge per one turn). Let $H_{n}$ be the graph that we get from $K_{n-2,2}$ by joining the two vertices in its two-element color class, see Figure 1.

Theorem 1. Playing the PrimMaker-Breaker game on a graph $G$ with $n$ vertices, PrimMaker wins as a first player if and only if $G$ contains $H_{n}$ as a subgraph.

It is interesting that in both directions of the proof of Theorem 1, the actual winner may utilize a pairing strategy. Breaker's strategy can be adapted to a $(1: b)$-game on $K_{n}$, and it shows that Breaker wins if $b>1$, in contrast to the probabilistic in- 
tuition which predicts $b_{0}=\Omega(n / \log n)$. As it was observed before, the acceleration of games has surprising effects [14], and it may restore the probabilistic intuition destroyed by a pairing strategy in the (1:1)-game [1]. Here we can witness, in magnitude, a perfect restoration of that intuition.

Theorem 2. Playing the (2:b) PrimMaker-Breaker game on $K_{n}$, Maker wins if $b<n /(8 \log n)$, and Breaker wins if $b>n / \ln n$.

\section{Background}

The following result is not just one of the most important results in the theory of hypergraph games, but it can be used very effectively to decide the winner of biased hypergraph games. The case $a=b=1$ was proved by Erdős and Selfridge in [8], and the general form was proved by Beck in [2].

Theorem 3. If

$$
\sum_{A \in E(\mathcal{H})}(1+b)^{-|A| / a}<1,
$$

then Breaker has a winning strategy in the $(\mathcal{H}, a, b)$ game.

However, several times not Theorem 3 but its proof techniques and corollaries are used.

For the sake of a better understanding and introducing some notations, we give a sketch of the proof of case $b=1$, and all elements of $E(\mathcal{H})$ have the same size, a more detailed proof can be found in [14].

The uniform case with $b=1$. For any $A \in V(\mathcal{H})$ let $A_{k}(M)$ and $A_{k}(B)$ be the number of elements in $A$, after Maker's $k$ th move, selected by Maker and Breaker, respectively. Now, for an $A \in E(\mathcal{H})$

$$
w_{k}(A)=\left\{\begin{array}{l}
\lambda^{A_{k}(M)} \text { if } A_{k}(B)=0 \\
0 \text { otherwise }
\end{array}\right.
$$

where $\lambda=2^{1 / a}$. For any $x \in V(\mathcal{H})$ let $w_{k}(x)=\sum_{x \in A} w_{k}(A)$. The numbers $w_{k}(A)$ and $w_{k}(x)$ are called the weight of $A$ and $x$ (in the $k$ th step), respectively.

In the $k$ th step Breaker chooses an unselected element $y^{k} \in V(\mathcal{H})$ of maximum weight. Setting $w_{k}=\sum_{A \in E(\mathcal{H})} w_{k}(A)$, called the potential, one gets $w_{k} \geq w_{k+1}$, $k \geq 0$.

In particular, $w_{1} \leq\left(\lambda^{a}-1\right)|E(\mathcal{H})|+|E(\mathcal{H})| \leq 2|E(\mathcal{H})|$. Since $b=1$ and the elements of $E(\mathcal{H})$ are of the same size, the inequality $\sum_{A \in E(\mathcal{H})} 2^{-|A| / a}<1 / 2$ leads to the inequality $2|E(\mathcal{H})|<2^{|A| / a}$. Let us suppose that Maker wins the game in the $k$ th step. This would imply that $w_{k} \geq \lambda^{|A|}=2^{|A| / a}$, contradicting the monotonicity of the potential.

An edge $A \in E(\mathcal{H})$ is active if Breaker has not taken any of its elements. Conversely, $A \in E(\mathcal{H})$ is blocked if Breaker has already taken an element of it. 
Since $w_{k} \leq w_{1} \leq 2|E(\mathcal{H})|$ for all $k$, we have a bound on the "fill-in" of an active edge. Note that this bound holds for the non-uniform hypergraphs as well.

Corollary 1. [14] Playing the Maker-Breaker $(\mathcal{H}, a, 1)$ game, Breaker may arrange that whenever $A$ is active, i.e. $A_{k}(B)=0$, then $A_{k}(M) \leq a+a \log _{2}|E(\mathcal{H})|$.

Proof of Corollary 1. Just take the logarithm of the inequality $\lambda^{A_{k}(I)}=w_{k}(A) \leq$ $w_{k} \leq w_{1} \leq 2|E(\mathcal{H})|$ that holds for any active edge $A \in E(\mathcal{H})$.

\section{Proofs}

Proof of Theorem 1. First we show if a graph $G$ on $n$ vertices contains the subgraph $H_{n}$, then PrimMaker wins the game as a first player. PrimMaker might restrict his moves to the edges of $H_{n}$ as follows. His first move is the edge $e=(u, v)$, the edge added to $K_{n-2,2}$, see Figure 1 . The other edges of $H_{n}$ are paired such that $f, g \in E\left(H_{n}\right) \backslash\{e\}$ is a pair if they are incident and their common endpoint lies in $V\left(H_{n}\right) \backslash\{u, v\}$. PrimMaker plays according to this pairing; more precisely, in every turn, he takes one element of a pair. This keeps his subgraph connected and results in a spanning tree in the $(n-1)$ st move.

In the other direction, let us assume that $G$ does not contain $H_{n}$, and PrimMaker's first move is an edge $e=(u, v)$. Then there must be a vertex $x \in$ $V(G) \backslash\{u, v\}$, such that $|N(x) \cap\{u, v\}| \leq 1$. Now Breaker might also use a pairing strategy: whenever PrimMaker connects a new vertex $y$ to his subgraph, i.e. takes an edge $(z, y)$, where $z$ had been visited earlier, Breaker takes the edge $(y, x)$ if $(y, x) \in E(G)$, and moves arbitrarily otherwise. Obviously, PrimMaker can never connect the vertex $x$ to his subgraph.

Note that we have proved a little more than was stated in Theorem 1. By winning PrimMaker builds a subgraph of diameter not more than three, which type of games was explored in [1].

Proof of Theorem 2. PrimMaker's win. First, we describe the winning strategy, and then show its feasibility. PrimMaker plays an equivalent auxiliary game, called the positive minimum degree game (see Hefetz et al. [10]), with the additional requirement that his subgraph should be connected during the game.
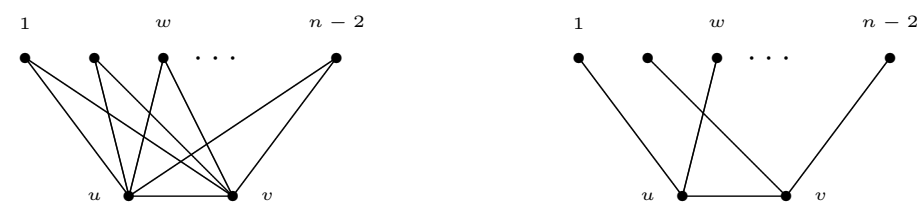

Figure 1: The graph $H_{n}$ and a possible Maker's subgraph. 
PrimMaker tries to get edges incident to each vertices as quickly as possible. More precisely, he can guarantee an edge incident to the vertex $x$, before Breaker takes, say, $n / 4$ edges incident to $x$. This can be achieved by an appropriate weight function method used several times before $[13,14,15]$.

In order to utilize Corollary 1, we associate an auxiliary hypergraph game with the PrimMaker-Breaker game. For each vertex $x \in V\left(K_{n}\right)$, let the $A_{x} \in E(\mathcal{H})$ be the set of ordered pairs $\langle x, y\rangle$, where $y \in V\left(K_{n}\right) \backslash x$. That is, $A_{x} \cap A_{y}=\emptyset$ for $x \neq y$ and $\left|A_{x}\right|=n-1$ for all $x \in V\left(K_{n}\right)$. When Maker takes the edge $(x, w)$ in the graph game, it results in taking both $\langle x, y\rangle$ and $\langle y, x\rangle$ in the hypergraph game. Of course Breaker's one move means taking $2 b$ ordered pairs. Note that PrimMaker intends to play as Breaker in this auxiliary game.

Let us assume that PrimMaker can imitate the greedy strategy of Corollary 1 in the $(\mathcal{H}, 1,2 b)$ game. Note that in order to do so, PrimMaker does not have to take the pair (edge) $\langle x, y\rangle$ of the largest weight, as taking any pair from the largest weight hyperedge has the same effect on the potential function $w_{k}$.

Extending the notation of Corollary 1, we may say that a vertex $x$ is blocked if $A_{x}$ is blocked, i.e. PrimMaker has an edge that is incident to $x$. We shall prove by induction on the steps of the game that an arbitrary vertex can be blocked at each step. The induction hypothesis holds in the first step, and assuming it holds up to the $k$ th step, we can use the bound of Corollary 1. This tells us that Breaker can take at most $b+b \log _{2} n \leq n / 4$ edges that are incident to an unblocked vertex $x$. Note that we can also assume that PrimMaker's edges form a tree $T_{i}$ after the $i$ th step, and $i \leq n / 2$. Indeed, in the process of blocking we never need to create cycles, so $\left|V\left(T_{i}\right)\right|=2 i+1$ if the game is not already over.

Let $T_{k}$ be PrimMaker's graph and $U_{k}$ be the set of unconnected (unblocked) vertices by PrimMaker after the $k$ th round, respectively. Assume that the blocking strategy requires one to block (connect) the vertex $x \in U_{k}$ in the $(k+1)$ th step. If there is an unoccupied edge $e=(x, y), y \in T_{k}$, then we take it. Similarly, if there are unoccupied edges $e=(x, y)$ and $f=(y, z), z \in T_{k}$ then we take those, and $x$ is blocked.

Assume on the contrary that there is a vertex $x \in U_{k}$ that cannot be blocked by PrimMaker in the $(k+1)$ st step; that is, in the subgraph of unoccupied edges there are no paths of length at most two from $x$ to $T_{k}$. According to the induction hypothesis, we know that Breaker has taken fewer than $n / 4$ edges incident to $x$. The other endpoints of these edges cannot be in $T_{k}$ and actually all the edges between these endpoints and the vertices of $T_{k}$ are taken by Breaker. The number of these edges is at least $(n-1-n / 4)(2 k+1)<3 n k / 2$, since $k \leq n / 2$. After round $k$, Breaker has claimed $b k$ edges, therefore we should have $3 n k / 2 \leq b k$, which contradicts the choice of $b$.

Breaker's win. This direction could be deduced from the results of Chvátal and Erdös, the only difference being that they examine $(1: b)$-game. For the sake of completeness, we sketch their proof. Breaker distributes his moves evenly. First, he puts an edge incident to all vertices, which needs no more than $n /(2 b)$ rounds. During that time PrimMaker may achieve a positive degree of at most $n / b$ vertices; 
these are dead for Breaker, while the others are active. Let us call the sequence of rounds a phase if Breaker gets a new incident edge to each (active) vertices. Breaker repeats the process above, getting a second, third, etc. edge incident to the edges that were all active at the beginning of the phase. Breaker loses the $n(1-1 / b)^{i} \geq 1$ fraction of active vertices, so the $i$ th phase is feasible if $n(1-1 / b)^{i} \geq 1$. That is, Breaker can reach the $n$th phase if $b>n / \ln n$. But by doing so, Breaker isolates a vertex.

\section{Further problems}

One could investigate PrimMaker-Breaker versions of any graph games, when Maker's present strategies involves disconnected edges. Sometimes the restriction of Breaker's move, introducing the notion of PrimBreaker seems to be a good idea.

Another possible way of defining a new game is to consider the game on a random graph, first investigated by Stojaković and Szabó [17]. For example if $V\left(\mathcal{H}_{p}\right)=E(G)$, where $G \in G(n, p)$ and the winning sets are the spanning trees of $G$. It was shown by Stojaković and Szabó that the probabilistic intuition applies to many of the random games, especially when there is a $p_{\mathcal{H}}$ such that if $p>p_{\mathcal{H}}$ then Maker, and if $p<p_{\mathcal{H}}$ then Breaker wins almost surely. The value of $p_{\mathcal{H}}$ should be close the threshold value of the connectivity of $G(n, p)$.

Needless to say, the PrimMaker-Breaker version of the random Shannon's switching game again defies random intuition. It would be interesting to see whether the $(2: 2)$ PrimMaker-Breaker version restores it. The proof method of Theorem 2 gives only $p_{\mathcal{H}} \leq c \log n / \sqrt{n}$, although $p_{\mathcal{H}} \leq c \log n / n$ would be desirable. However, one might argue that $(2: 2)$ acceleration is not enough, and $(3: 3)$, or even more is needed. Another possible line is to define the PrimMaker-PrimBreaker version where the restriction of Breaker brings $p_{\mathcal{H}}$ closer to the $c \log n / n$ bound.

\section{References}

[1] Balogh, József, Martin, Ryan, and Pluhár, András. The diameter game. Random Structures \&3 Algorithms, 35(3):369-389, 2009.

[2] Beck, József. Remarks on positional games. Acta Mathematica Hungarica, 40(1-2):65-71, 1982.

[3] Beck, József. Deterministic graph games and a probabilistic intuition. In Combinatorics, Geometry and Probability, a Tribute to Paul Erdös, pages 8194. Cambridge University Press, 1997.

[4] Beck, József. Combinatorial games: tic-tac-toe theory. In Encyclopedia of Mathematics and its Applications, volume 114. Cambridge University Press, 2008. 
[5] Bednarska, Małgorzata and Łuczak, Tomasz. Biased positional games for which random strategies are nearly optimal. Combinatorica, 20(4):477-488, 2000 .

[6] Bollobás, Béla. Random graphs. In Modern Graph Theory, pages 215-252. Springer, 1998.

[7] Chvátal, Vašek and Erdős, Paul. Biased positional games. Annals of Discrete Mathematics, 2:221-229, 1978.

[8] Erdős, Paul and Selfridge, John L. On a combinatorial game. Journal of Combinatorial Theory, Series A, 14(3):298-301, 1973.

[9] Espig, Lisa, Frieze, Alan, Krivelevich, Michael, and Pegden, Wesley. Walkerbreaker games. SIAM Journal on Discrete Mathematics, 29(3):1476-1485, 2015.

[10] Hefetz, Dan, Krivelevich, Michael, Stojaković, Miloš, and Szabó, Tibor. Global maker-breaker games on sparse graphs. European Journal of Combinatorics, 32(2):162-177, 2011.

[11] Kruskal, Joseph B. On the shortest spanning subtree of a graph and the traveling salesman problem. Proceedings of the American Mathematical society, $7(1): 48-50,1956$.

[12] Lehman, Alfred. A solution of the Shannon switching game. Journal of the Society for Industrial and Applied Mathematics, 12(4):687-725, 1964.

[13] Pluhár, András. Generalized Harary games. Acta Cybernetica, 13(1):77-83, 1997.

[14] Pluhár, András. The accelerated k-in-a-row game. Theoretical Computer Science, 270(1-2):865-875, 2002.

[15] Pluhár, András. The recycled Kaplansky's game. Acta Cybernetica, 16(3):451458, 2004.

[16] Prim, Robert Clay. Shortest connection networks and some generalizations. Bell Labs Technical Journal, 36(6):1389-1401, 1957.

[17] Stojaković, Miloš and Szabó, Tibor. Positional games on random graphs. Random Structures \& Algorithms, 26(1-2):204-223, 2005. 